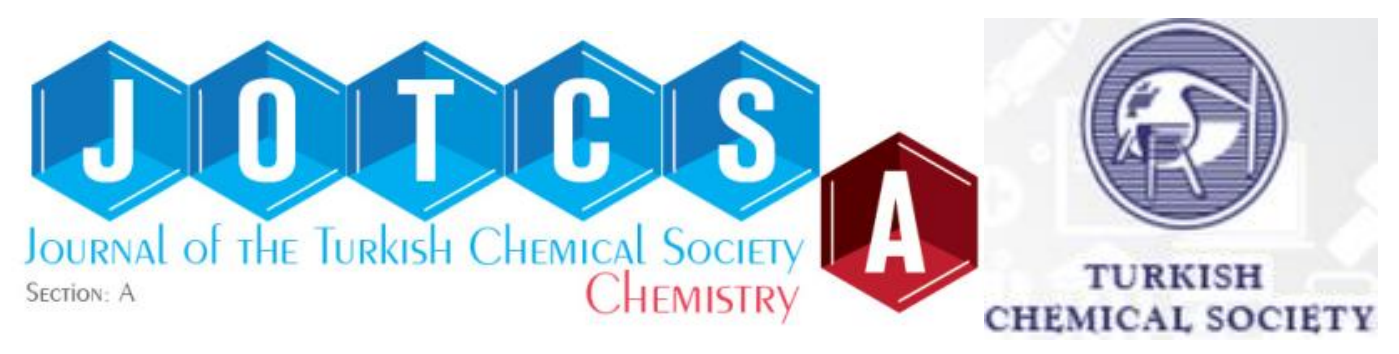

\title{
PVA NANOCOMPOSITES OF ORGANOCLAYS OBTAINED USING DIFFERENT CATIONIC SURFACTANTS
}

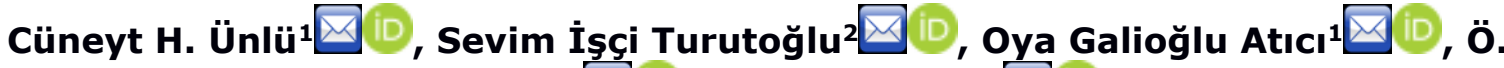 Işık Ece ${ }^{3}$, Nurfer Güngör ${ }^{2}$ (D)}

${ }^{1}$ Istanbul Technical University, Science \& Letters Faculty, Chemistry Department, Maslak 34469, Istanbul, TURKEY. ${ }^{2}$ Istanbul Technical University, Science \& Letters Faculty, Physics Department, Maslak 34469, Istanbul, TURKEY. ${ }^{3}$ Istanbul Technical University, Faculty of Mines, Department of Geology, Maslak 34469, Istanbul, TURKEY

\begin{abstract}
This study is about the preparation of two different organoclays with cationic surfactants and their poly(vinyl alcohol) nanocomposites with increased thermal and mechanical behavior. Organoclays were prepared to modify clay mineral with solution intercalation method using aqueous solutions of cationic surfactants dodecyltrimethylammonium bromide (DTABr) and cetylpyridinium bromide (CPBr). Obtained organoclays (D-MMT and C-MMT for DTABr/MMT and CPBr/MMT, respectively) were characterized using different methods including zeta potential and XRD. Results indicated an absolute decrease in zeta potential about $20 \mathrm{mV}$ for C-MMT and $14 \mathrm{mV}$ for D-MMT indicating flocculation and coating of the surface. Moreover, measurements indicated that interlayer distance increased based on basal spacing peak shift whose value was $1.27 \mathrm{~nm}$ for NaMMT, whereas $1.40 \mathrm{~nm}$ for D-MMT, and $1.75 \mathrm{~nm}$ for C-MMT. The organoclays were used in the preparation of PVA/clay nanocomposites; thermal stability of the nanocomposites was determined using TGA, while mechanical strength measurements were done using DMA. The maximum thermal decomposition temperature of the pristine PVA and nanocomposites were compared, and an average increase of $4{ }^{\circ} \mathrm{C}$ was observed. Also, the activation energy of the decomposition was observed ca. 40 $\mathrm{kJ} \cdot \mathrm{mol}^{-1}$ higher than pristine PVA.
\end{abstract}

Keywords: Polymer/clay nanocomposites, Organoclay, Poly(vinyl alcohol) (PVA), Thermal stability, Cationic surfactants, Principal components analysis.

Submitted: April 22, 2017 . Accepted: February 20, 2018.

Cite this: Ünlü $C$, Turutoğlu $S$, Galioğlu Atıcı O, Ece Ö, Güngör N. PVA NANOCOMPOSITES OF ORGANOCLAYS OBTAINED USING DIFFERENT CATIONIC SURFACTANTS. JOTCSA. 2018;5(2):415-32.

DOI: http://dx.doi.org/10.18596/jotcsa.307602.

*Corresponding author. E-mail: unlucu@itu.edu.tr, fax:+90-212-285 6386. 


\section{INTRODUCTION}

One of the popular research topics is polymer/clay nanocomposites and has been studied extensively in recent years.(1-5) Research in this field has been started with Toyota R\&D department's work on nylon in the early nineties in order to produce materials with enhanced physical and chemical properties. Later, other applications of polymer/clay nanocomposites in different industrial fields are also developed such as food packaging, automobile parts, microelectronic packaging, and medical tubing.(5-7) Montmorillonite, which is a member of the smectite group clay minerals, is the most popular material used in polymer/clay nanocomposites. Smectites are composed of approximately $1 \mathrm{~nm}$ thick layers with high aspect ratios; each layer consists of one alumina sheet between two silica sheets. Due to naturally occurring network defects, large surfaces of the layers (faces) bear negative charges, and these faces can interact electrostatically with inorganic (exchangeable) cations such as sodium, potassium, calcium which reside among interlayer galleries of the clay mineral. When clay mineral swells in water interlayer galleries expand and the exchangeable cations substitute with organic (i.e., surface active agents or polymeric materials) compounds to form intercalated compounds resulting in an increase in basal spacing of the clay. In some cases, the polymeric matrix may separate the clay layers forming an exfoliated nanocomposite.(8) As the clay surfaces have hydrophilic nature, modifying them to obtain organophilic surfaces with the use of cationic surfactants for exchangeable cations may help penetration of the polymeric material into the galleries finally producing a better and uniformly dispersed clay mineral in the polymeric matrix. Alkylammonium salts are the most used cationic surfactant for production of organoclays.(9)

Poly(vinyl alcohol), PVA, is a cheap, water soluble, and non-toxic polymeric material and has many applications in different industrial areas including textiles, and coating. $(10,11)$ In polymer/clay nanocomposites prepared with PVA polymer matrix may be solely PVA or along with other polymeric materials (such as cellulose, chitosan) in order to enhance material properties. (11-15) Addition of clay into PVA matrix causes an increase in mechanical and thermal properties as expected as well as enhancements in film formation capability and chemical resistance. Recent studies with PVA indicate that organically modified montmorillonite layers more easily disperse into the PVA matrix uniformly producing an exfoliated nanostructures.

The aim of this study can be divided into two subsections; the first part is the synthesis of organoclays by modification with use of two different cationic surfactants; then the 
second part is the formation of PVA/organoclay nanocomposites using organoclays via the solution intercalation method. The results are evaluated using different characterizations methods such as spectroscopy (FTIR, X-ray diffraction), dynamic mechanical analysis (DMA), and thermogravimetric analysis (TGA).

\section{EXPERIMENTAL SECTION}

\section{Materials and Methods}

Poly(vinyl alcohol) (PVA, molecular weight $145 \mathrm{~kg} / \mathrm{mol}$ ), dodecyltrimethylammonium bromide (DTABr, $\quad \mathrm{C}_{12} \mathrm{H}_{25} \mathrm{~N}\left(\mathrm{CH}_{3}\right)_{3} \mathrm{Br}$ ) and cetylpyridinium bromide ( $\mathrm{CPBr}$, $\left.\mathrm{C}_{5} \mathrm{H}_{5} \mathrm{NCH}_{2}\left(\mathrm{CH}_{2}\right)_{14} \mathrm{CH}_{3}\right)$ were Fluka brand; they were used as received.

The clay mineral was Na-montmorillonite (NaMMT) collected locally from Enez-Turkey. The dominant clay mineral was found to be dioctahedral montmorillonite with minor amounts of illite and kaolinite. The chemical composition of NaMMT was determined by atomic adsorption spectroscopy (Perkin Elmer 3030 model), and the silica content was determined gravimetrically. The chemical composition of clay sample was (wt.\%): $\mathrm{SiO}_{2}$ 58.8, $\mathrm{Al}_{2} \mathrm{O}_{3}$ 18.73, $\mathrm{Fe}_{2} \mathrm{O}_{3}$ 3.71, $\mathrm{CaO} 3.34, \mathrm{MnO} 0.09, \mathrm{Na}_{2} \mathrm{O} 3.36, \mathrm{MgO} 2.62, \mathrm{~K}_{2} \mathrm{O} 2.70$ and $\mathrm{TiO}_{2} 0.49$.

Cation exchange capacity (CEC) of clay mineral was determined using methylene blue method.(16) Methylene blue solution was prepared and added to clay dispersion in small amounts. Then a droplet of the dispersion was placed on a filter paper. When a blue circle around the blot was observed this meant that the clay was saturated with the dye thus releasing an excess of it. CEC for NaMMT was determined as 89 milliequivalents per $100 \mathrm{~g}$ clay.

\section{Characterization Methods}

The zeta potential measurements were carried out using Malvern Instruments Zetasizer 2000 with an optical unit which had $5 \mathrm{~mW}$ He-Ne $(638 \mathrm{~nm}$ ) laser. All dispersions were centrifuged at $6000 \mathrm{rpm}$ for 30 minutes before the measurements then supernatant fraction was used for the measurements.

X-ray diffraction (XRD) analyses of solvent-cast films were performed on Philips PW 1040 at room temperature using $\mathrm{Ni}$-filtered $\mathrm{Cu}-\mathrm{K}_{\mathrm{a}}$ radiation. The diffractograms were obtained by scanning in $2 \theta$ ranges from 2 to $10^{\circ}$ at a rate of 2 degrees per minute. 
FTIR analyses were done on Perkin Elmer Spectrum One FTIR spectrophotometer in $4000-400 \mathrm{~cm}^{-1}$ range as either $1 \% \mathrm{KBr}$ pellet or solvent-cast film; results were recorded in absorbance mode.

Principal component analysis (PCA) of the FTIR data was performed using R (version 3.4.3) software. First, the data were processed to obtain derivative spectra (first and second order) within a selected range (1800-400 cm-1) using Savitzky-Golay procedure. Then PCA was applied to the data in order to see clustering.

Dynamic mechanical analyses (DMA) were performed on Perkin Elmer Diamond DMA under tension film mode in a temperature range $20-200^{\circ} \mathrm{C}$ at a frequency of $1 \mathrm{~Hz}$ and a heating rate of $5^{\circ} \mathrm{C} / \mathrm{min}$. Samples were prepared by the solvent casting method.

The thermal properties were analyzed by thermogravimetric analysis (TGA) on TA Instruments $\mathrm{Q} 50$ with a heating rate of $20^{\circ} \mathrm{C} / \mathrm{min}$ under $\mathrm{N} 2$ atmosphere in a range of 25-600 ${ }^{\circ} \mathrm{C}$. Kinetic parameters were calculated using Broido method(17) with Python interpreter NumPy and SciPy modules.

\section{Preparation of Organoclay}

The organoclay as prepared by using cationic surfactants as intercalating agents. NaMMT $(10 \mathrm{~g})$ was dispersed in $500 \mathrm{ml} 50 \%$ aqueous ethanol at $65^{\circ} \mathrm{C}$. Then the surfactant $(0.01$ $\mathrm{M}$, $\mathrm{DTABr}$ or $\mathrm{CPBr}$ ) was added; $\mathrm{pH}$ of the dispersion was adjusted to 3.5 with aqueous $\mathrm{HCl}$. The dispersion was stirred at $65^{\circ} \mathrm{C}$ for 1 hour then samples were centrifuged; obtained organoclay (D-MMT or C-MMT) was washed two times with water at $70^{\circ} \mathrm{C}$ and dried at $40^{\circ} \mathrm{C}$.

\section{Preparation of PVA/Organoclay Nanocomposites}

Aqueous organoclay dispersion ( $1 \%$ by weight) was shaken for 2 days in order to obtain uniformly and well-swelled organoclay. Then an appropriate amount of the dispersion was blended with $5 \mathrm{~g}$ PVA in $200 \mathrm{ml}$ distilled water during 100 minutes at $75( \pm 5){ }^{\circ} \mathrm{C}$. Obtained PVA/organoclay nanocomposites had organoclay contents of at 1,2 , and $3 \%$ by weight with respect to PVA. The products dried at room temperature and named as DPVA or C-PVA denoting the surfactant used was D-MMT or C-MMT, respectively. 


\section{RESULTS AND DISCUSSION}

\section{Characterization of Clay and Organoclay Dispersions}

Zeta potential characterization techniques rely on the determination of total surface charge of the colloidal particles. The instrument uses a laser beam to count the number of the charged particles moving between oppositely charged electrodes under a potential difference. Surface charge (or zeta potential) may differ if coagulation or flocculation occurs because the size of the particle grows while charges on the surface may decrease or increase as a result of the interactions. Clay particles carry a net surface charge due to the structure of the clay; larger siloxane surfaces (face, Si-O-Si groups) are negatively charged due to substitutional point defects in the lattice while smaller side surfaces (edges, aluminol, $\mathrm{Al}-\mathrm{OH}$ and silanol, $\mathrm{Si}-\mathrm{OH}$ groups) have positive charges. Electrostatic interactions among oppositely charged clay surfaces cause screening of the charges and lead to flocculation. This occurrence results in a net decrease of the surface charge (regardless of the sign) of the large clay particles. Interactions may take place among edges and faces at different aspects (edge-face, edge-edge, and face-face) resulting in variations in shapes and sizes of the particles.(18)

The zeta potential of NaMMT dispersion was measured as $-41.20 \mathrm{mV}$ indicating the dispersion had deflocculated structure. However, zeta potential values belonging to organoclays were found as -27.6 for D-MMT and $-22.23 \mathrm{mV}$ for C-MMT. The absolute decrease in zeta potentials of the organoclay dispersions was the indicator of claysurfactant interactions; surfactant molecules covered surfaces of the clay particles leading to flocculation. According to the results, especially C-MMT had a more flocculated structure in the dispersion compared to D-MMT.

Basal spacing ( $d_{001}$ ), another key feature directly related to the structure, was measured using XRD. Diffractograms of NaMMT, C-MMT, and D-MMT were shown in Figure 1. The values indicated some intercalation for both organoclays. NaMMT had a doo1 value of $1.271 \mathrm{~nm}$. After modification of NaMMT, this value increased to $1.401 \mathrm{~nm}$ for D-MMT and $1.748 \mathrm{~nm}$ for C-MMT. These increases displayed that clay layers were separated slightly as a result of intercalation by cationic surfactants, but in different amounts. This occurrence might be related to the orientation of the surfactants into the interlayer galleries. The basal spacing increase was found greater for C-MMT $(0.48 \mathrm{~nm})$ than DMMT $(0.13 \mathrm{~nm})$ probably due to bilayer type arrangement of the $\mathrm{CPBr}$ molecules parallel to the silicate layer.(19) 


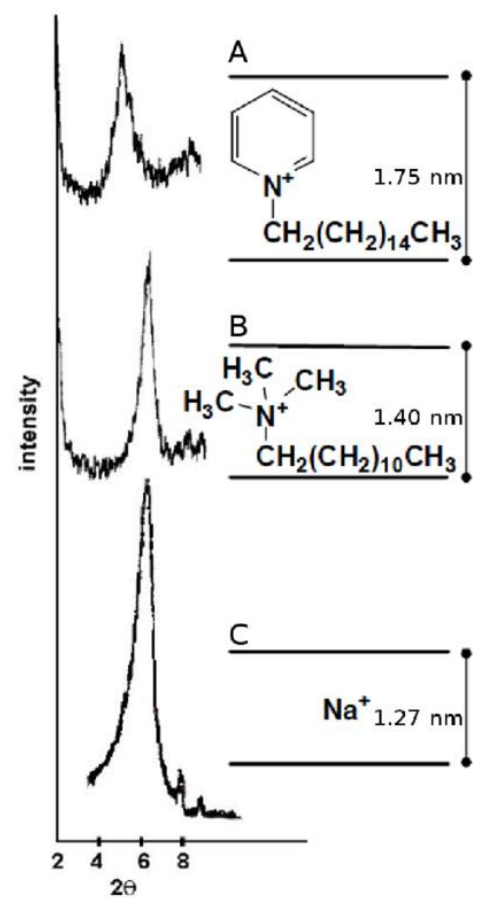

Figure 1. XRD diffractograms of C-MMT (a), D-MMT (b), and NaMMT (c).

Better intercalation of NaMMT by $\mathrm{CPBr}$ was also attributed to alkyl chain length of cationic surfactants, which had a positive effect on adsorption by clays; longer chain caused an increase in adsorption by clay surface via Van der Waals forces.(20-22) The mentioned interactions among surfactant and clay were stronger for $\mathrm{CPBr}$ compared to $\mathrm{DTABr}$ under same conditions. Finally, another plausible cause for this result was related with aqueous solubilities of the surfactants; $\mathrm{DTABr}$ was more soluble in water compared with $\mathrm{CPBr}$; thus desorption of $\mathrm{DTABr}$ from clay surfaces to aqueous phase would be higher than $\mathrm{CPBr}$. Once $\mathrm{CP}^{+}$cation was adsorbed by the clay surface, it hardly detached and went back to the aqueous phase, unlike DTA ${ }^{+}$cation.

Evaluation of the results obtained from XRD and electrokinetic measurements indicated that (a) surfactant molecules were adsorbed by both inner and outer surfaces of the clay, and (b) CPBr intercalated NaMMT more efficiently compared to DTABr.

\section{Characterization of PVA/Organoclay Nanocomposites}

The PVA/organoclay nanocomposites (C-PVA and D-PVA) were identified using several spectroscopic techniques. XRD measurements of both nanocomposites with different clay concentrations were done to observe intercalation of PVA into interlayer galleries of the organoclay. Basal spacing peak (d $\mathrm{d}_{001}$ ) of NaMMT was absent in all diffractograms of PVA nanocomposites (Figure 2). The disappearance of the do01 peak might be attributed to the shift of this peak under $2^{\circ}$ diffraction angle due to highly intercalated or completely 
exfoliated organoclay platelets. As literature survey about PVA/(organo)clay nanocomposites indicated that this occurrence was highly plausible.(14) Thus

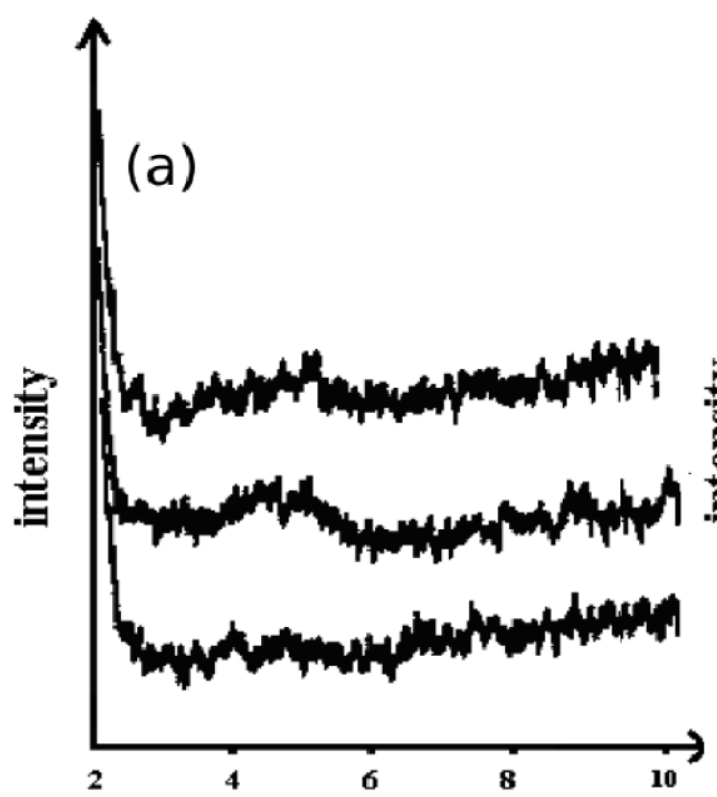

$2 \theta$

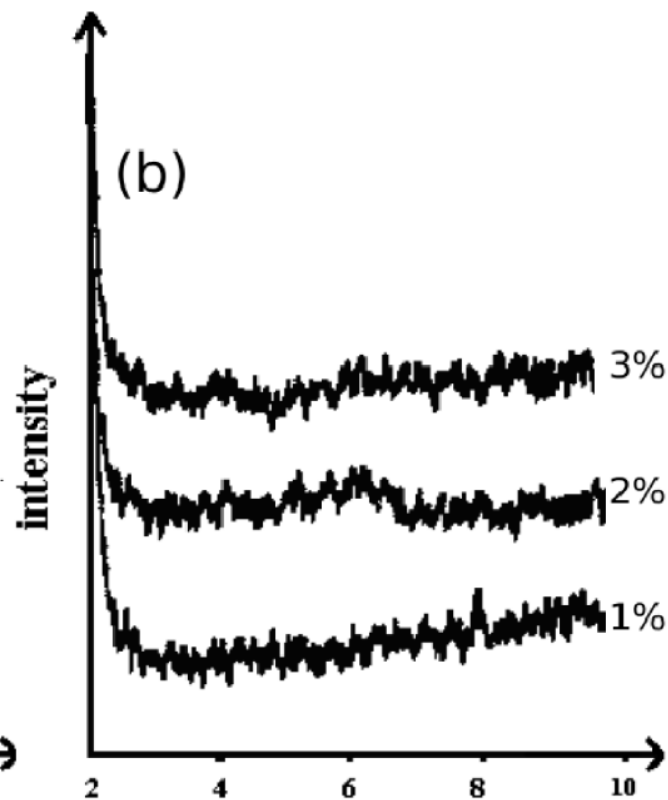

$2 \theta$

intercalated platelets possibly dispersed within PVA matrix forming nanostructures.

Figure 2. XRD diffractograms of D-PVA (a), and C-PVA (b).

The structure of the PVA nanocomposites was also analyzed using FTIR spectroscopy. FTIR spectra of PVA, NaMMT, and nanocomposites were first evaluated separately then compared with each other. Characteristic vibrations and attributed vibration modes for pristine PVA were observed as $3339 \mathrm{~cm}^{-1}$ (O-H stretching), 2942, $2912 \mathrm{~cm}^{-1}$ (alkyl C-H stretching), 1436, $1424 \mathrm{~cm}^{-1}$ (C-H bending), $1093 \mathrm{~cm}^{-1}$ (C-O stretching). Signals related to residual acetate groups in the polymer were observed at $1707 \mathrm{~cm}^{-1}$ ( $C=O$ stretching with intramolecular $\mathrm{H}$-bond), $1377 \mathrm{~cm}^{-1}\left(\mathrm{CH}_{3}\right.$ group) and $1238 \mathrm{~cm}^{-1}$ (C-O bending).(23)

Characteristic absorption bands of NaMMT were determined as structural $\left(3627 \mathrm{~cm}^{-1}\right)$ and intra/intermolecular hydrogen bonded $\mathrm{O}-\mathrm{H}$ stretching $\left(3453 \mathrm{~cm}^{-1}\right), \mathrm{H}-\mathrm{O}-\mathrm{H}$ deformation vibration due to adsorbed water $\left(1635 \mathrm{~cm}^{-1}\right)$, Si-O stretching vibration at $1033 \mathrm{~cm}^{-1}$ with a shoulder at $1087 \mathrm{~cm}^{-1}$, Al-OH (917 and $622 \mathrm{~cm}^{-1}$ ) and (Al, Mg)-O (848 and $793 \mathrm{~cm}^{-1}$ ) vibrations modes, Si-O bending vibration at 521 and $467 \mathrm{~cm}^{-1} \cdot(24,25)$

Structural O-H vibration of MMT at $3627 \mathrm{~cm}^{-1}$ was absent in the spectra of organoclays; it possibly overlapped with $\mathrm{O}-\mathrm{H}$ stretching of PVA. The strongest signal of organoclay spectrum was $\mathrm{Si}-\mathrm{O}$ stretching at $1033 \mathrm{~cm}^{-1}$ and it also overlapped with $\mathrm{C}-\mathrm{O}$ stretching of PVA at $1093 \mathrm{~cm}^{-1}$ and could not be observed in the spectra of PVA/organoclay 
nanocomposites; however, its presence was slightly distinguishable by a widening towards $1050 \mathrm{~cm}^{-1}$ in C-O signal (Figure 3).

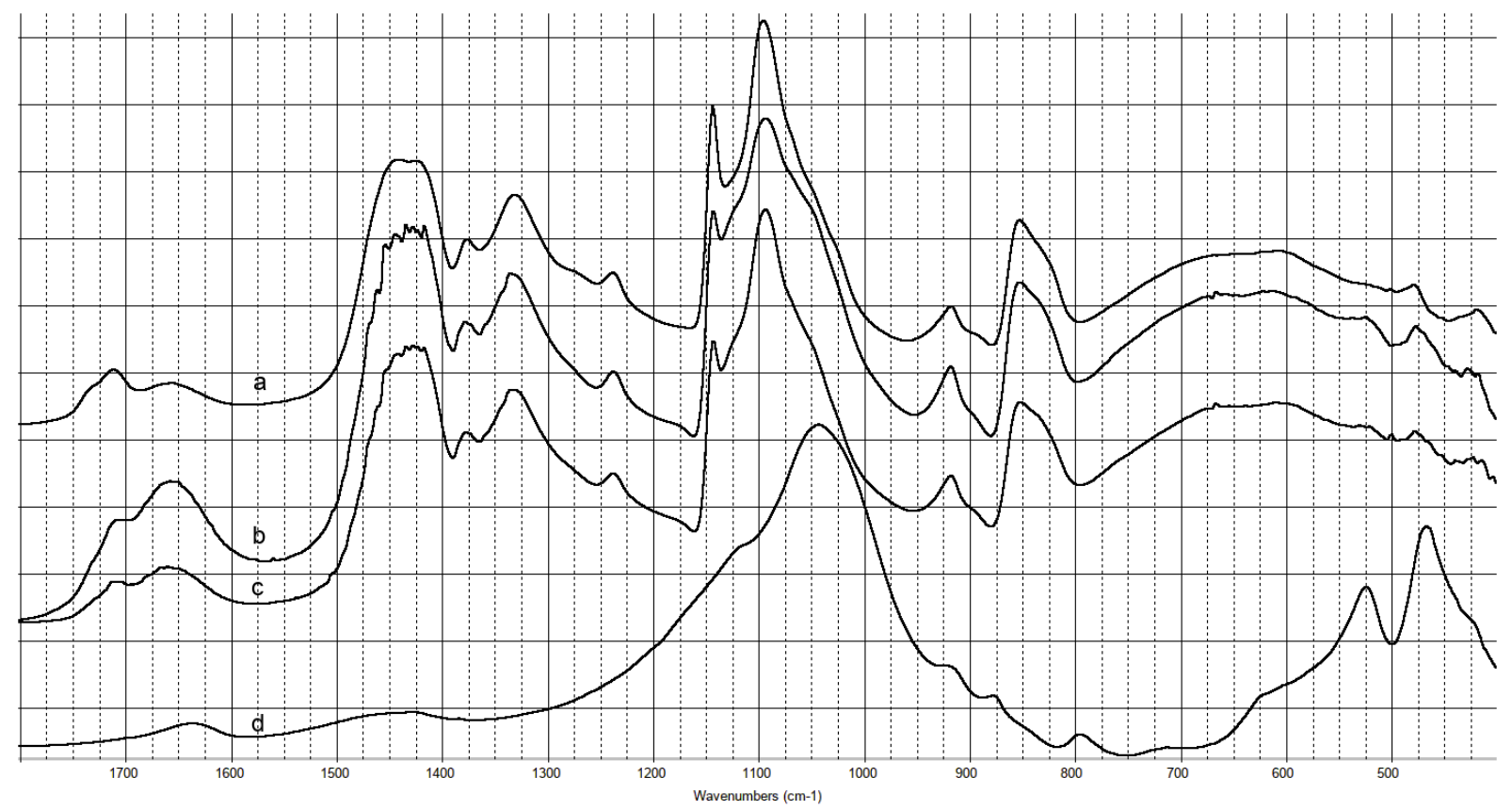

Figure 3. FTIR spectra of nanocomposites PVA (a), D-PVA (b), C-PVA (c) with $2 \%$ clay and pristine NaMMT (d).

The magnitude of the signal widening was directly proportional to the amount of organoclay in the PVA/organoclay system; the shoulder at $1054 \mathrm{~cm}^{-1}$ became more distinct as the amount of organoclay increased. Another factor affecting signal shape in this region was the type of the surfactant used in organoclay. The shape of the signal observed more distorted for D-PVA compared to C-PVA under same conditions indicating a difference in the interaction between organoclay and PVA. Another indicator of organoclay presence in PVA matrix was slightly increasing the intensity of signals belonging to $\mathrm{Si}-\mathrm{O}$ bending vibrations at 521 and $467 \mathrm{~cm}^{-1}$. Peak positions of these signals were slightly shifted according to organoclay type and amount in the PVA matrix. These results proposed that a change in crystal structure of the organoclay as a result of the interaction with PVA matrix.

As the difference is very hard to detect among the spectra, a statistical method called as principal component analysis (PCA) was used to emphasize the differences among the data. Differences among the spectra were observed mainly in range 1800-400 where characteristic absorption bands of PVA and MMT were located. As the signals of C-O stretching and bending signals of PVA were overlapping with Si-O stretchings of MMT, it was very hard to distinguish the interaction. Thus the first-order derivative of each sample was calculated using Savitzky-Golay algorithm. Then PCA was applied to these 
derivative data in R software with centering. Analysis of total the variance change yielded that the first three principal components (PCs) were responsible for $99.05 \%$ of the total change. Further analysis of the PCs indicated that PC1 was responsible for $92.48 \%$ of the total change, while PC2 and PC3 were $4.05 \%$ and $2.49 \%$, respectively. Thus plotting PC1 versus PC2 gave a meaningful comparison for the classification of the samples. Scores plot was shown in Figure 4a. As seen in the scores plot PVA and NaMMT located at opposite sides of the PC1. This occurrence was examined using loadings plot (Figure 4b). In the figure PC1, PC2 and derivatives of PVA and NaMMT were plotted with raw spectra at the bottom. PC1 and derivative PVA had the same signals at the same positions with the exception of the sign; thus PVA was located at the left-hand side of the PC1. However, derivative NaMMT and PC1 had an in-phase correlation placing the NaMMT on the positive side of the PC1.

As a result of the PCA analyses, PVA, NaMMT, and composites located in different parts of the scores plot indicating they had spectral features concerning derivative FTIR spectra. It could be concluded that the appearance of the samples at different portions of the scores plot was attributed to the change in the FTIR signals and it was related with the amount and type of the organoclay used.
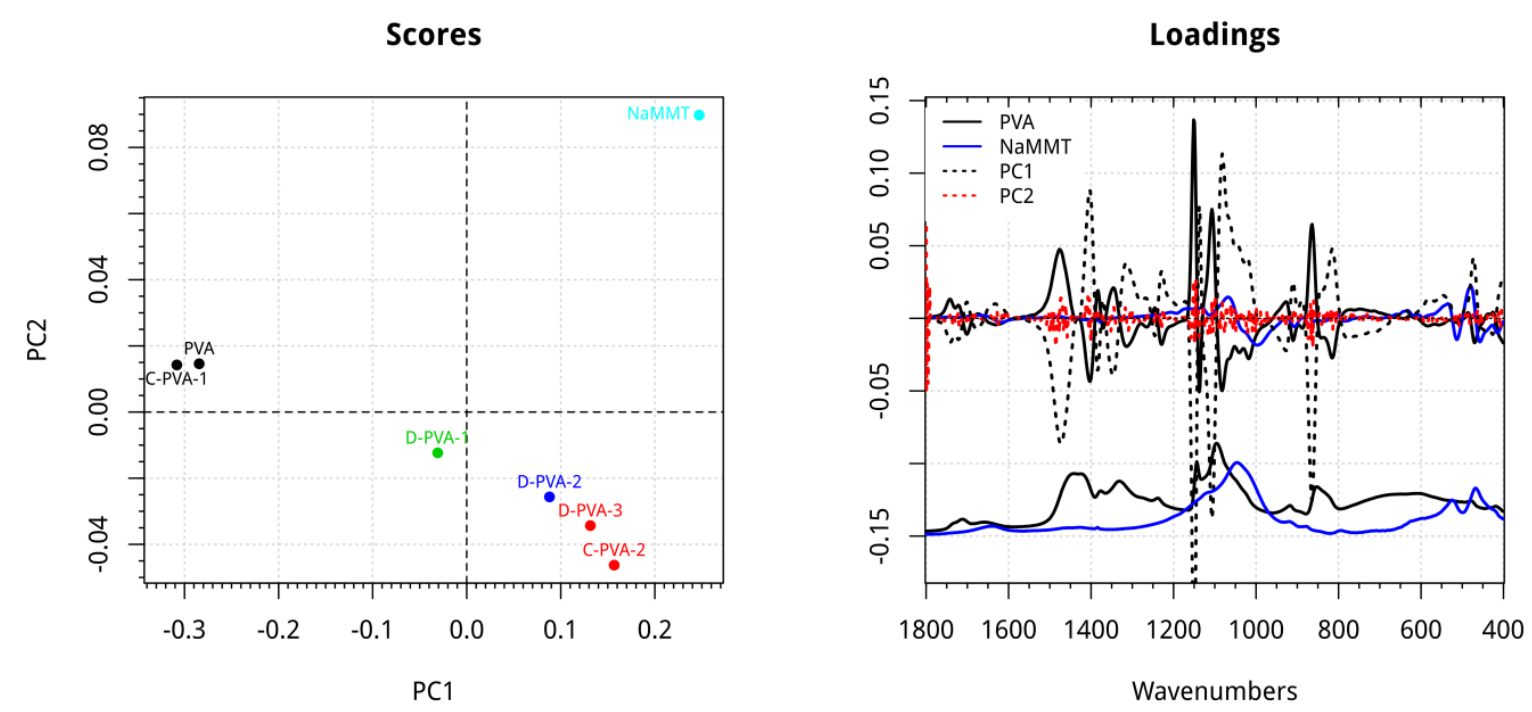

Figure 4. Scores plot of derivative FTIR spectra and loadings plot for PC1, PC2, PVA and NaMMT samples (lower part raw spectra of PVA and NaMMT).

Evaluation of XRD, FTIR and PCA analyses with literature data altogether lead to two results: (a) organoclays and PVA interacted possibly giving intercalated nanocomposites, and (b) type of the surfactant used played an important role on interaction efficiency, CMMT better than D-MMT regarding signal shifts. 


\section{Thermal and Dynamic Mechanical Characterization of PVA Nanocomposites}

Thermogravimetric analyses (TGA) were done to determine the contribution of organoclay to the thermal stability of the PVA; results of thermal analyses were summarized in Table 1. TGA thermograms, regardless pristine or organoclay containing PVA, showed about $7 \%$ weight loss starting from 80 to $200{ }^{\circ} \mathrm{C}$ which was attributed to water loss adsorbed by the sample (Figure 5). Char amount at the end was about $4.8 \%$ for PVA and D-PVA samples. However, C-PVA samples displayed lower char amount (2\%) compared with other samples.

Table 1. Results of TGA measurements and activation energy estimations regarding Broido method with correlation coefficients higher than 0.995 .

\begin{tabular}{lccccccc}
\hline \multicolumn{1}{c}{ Sample } & \multicolumn{2}{c}{ Clay } & \multicolumn{2}{c}{ Max. weight loss } & \multicolumn{4}{c}{ Activation energy $^{\mathbf{a}}$} \\
& $\mathbf{\%}, \mathbf{( w / w )}$ & At $\mathbf{3 8 0} \mathbf{\circ}^{\circ} \mathbf{C}$ & At $\mathbf{4 4 0}{ }^{\circ} \mathbf{C}$ & I & II & III & IV \\
\hline PVA & 0 & 377.5 & 440.7 & 86 & 133 & 51 & 84 \\
C-PVA & 1 & 382.6 & 444.4 & 117 & 177 & 69 & 120 \\
& 2 & 381.6 & 447.2 & 121 & 174 & 60 & 96 \\
& 3 & 381.8 & 449.9 & 126 & 176 & 61 & 98 \\
D-PVA & 1 & 380.1 & 440.8 & 127 & 162 & 62 & 103 \\
& 2 & 380.3 & 441.1 & 141 & 167 & 58 & 101 \\
& 3 & 378.4 & 441.3 & 130 & 170 & 63 & 111 \\
\hline
\end{tabular}

${ }^{\mathrm{A}} 300-350^{\circ} \mathrm{C}$, II $350-400^{\circ} \mathrm{C}$, III $400-440^{\circ} \mathrm{C}$, IV $440-470{ }^{\circ} \mathrm{C}$
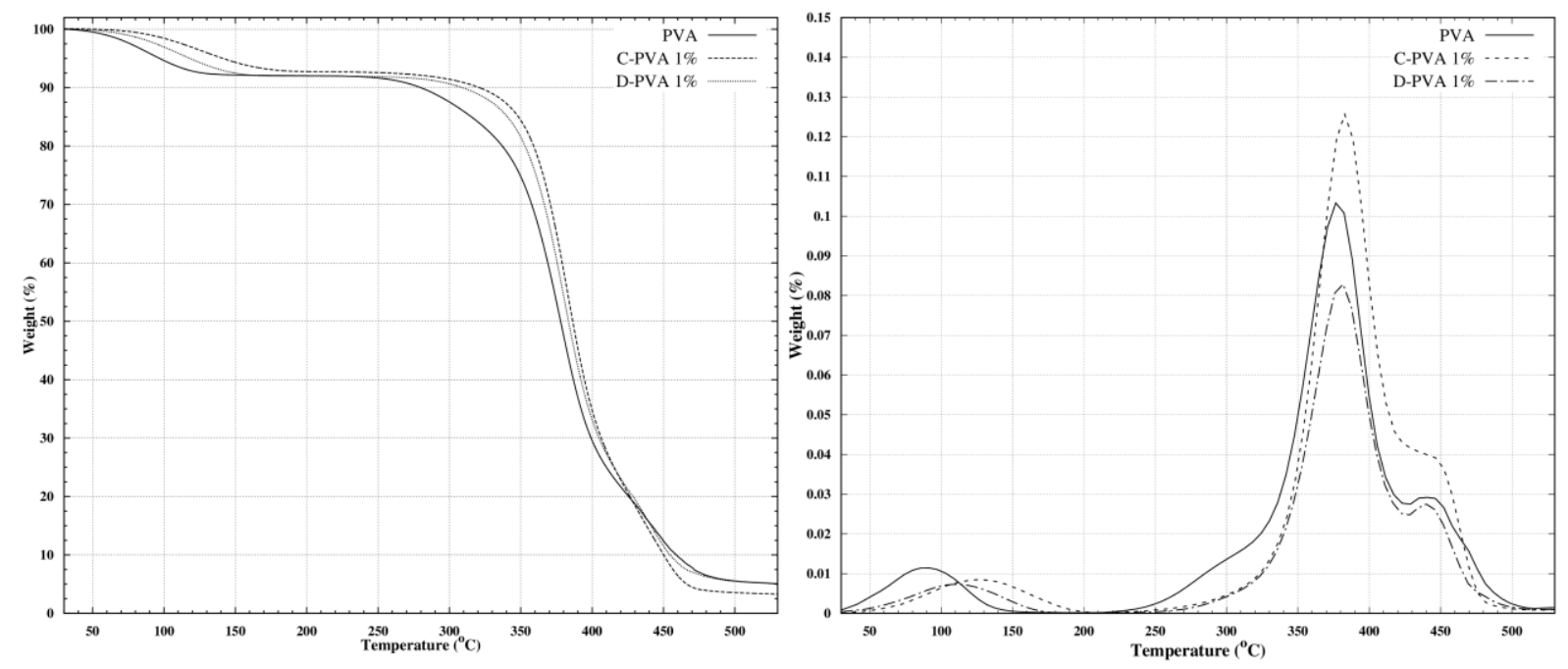

Figure 5. TGA (a) and derivative TGA (b) thermograms of PVA, $1 \%$ C-PVA, and D-PVA nanocomposites.

Maximum degradation temperature of the samples was determined using the first-order derivative of weight loss-temperature plot. In terms of degradation temperature pristine PVA displayed two maxima; a major signal $\left(\mathrm{T}_{1}\right)$ at $378^{\circ} \mathrm{C}$ as a peak, and a minor distinguishable signal $\left(\mathrm{T}_{2}\right)$ at $441^{\circ} \mathrm{C}$ as a shoulder. Thus thermal degradation kinetics of 
PVA could be separated two main parts; the first part was mainly related to dehydration and decarboxylation and second part covered more complex reactions and chain scissions.(26-29) The first part of the decomposition started around $300{ }^{\circ} \mathrm{C}$ which was observed as a shoulder in derivative TG plot (Figure $5 b$ ). This data was consistent with the literature cited.

Organoclay addition caused an increase in both degradation maxima of the PVA as an indicator of enhanced thermal stability. The degree of improvement was related to the organoclay used. In the case of C-PVA samples $\mathrm{T}_{1}$ position was observed at $382^{\circ} \mathrm{C}$, approximately $5^{\circ} \mathrm{C}$ higher compared to pristine PVA regardless of C-MMT content. In a similar manner $\mathrm{T}_{2}$ values were observed at higher temperatures; however, this time clay proportion caused some variations. $\mathrm{T}_{2}$ peak was at $444^{\circ} \mathrm{C}$ for C-PVA with $1 \% \mathrm{C}$-MMT content, $3{ }^{\circ} \mathrm{C}$ higher compared with pristine PVA; each $1 \%$ clay increase in C-MMT content caused an approximate increase of $3^{\circ} \mathrm{C}$. In the case of D-PVA a small increase in $\mathrm{T}_{1}$ was observed compared to pristine PVA with an approximate value of $2.5^{\circ} \mathrm{C}$ for 1 and $2 \% \mathrm{D}-\mathrm{MMT}$ content and ca. $1.0^{\circ} \mathrm{C}$ for $3 \% \mathrm{C}-\mathrm{MMT}$; however, $\mathrm{T}_{2}$ values were comparatively same regardless of clay content.

The detailed examination of the thermograms was done to find out decomposition activation energy values as a measure of thermal stability using Broido's method with Python SciPy and NumPy modules with a single run TGA measurement to evaluate the data. After the data acquisition some necessary manipulations were done to create the plot of the $\ln (\ln 1 / y)$ as a function of reciprocal temperature whose slope was the activation energy over universal gas constant where $y$ was the fraction at temperature $t$ regarding the remaining and starting weights of the decomposition $\left(\mathrm{W}_{\mathrm{t}}, \mathrm{W}_{\infty}, \mathrm{W}_{0}\right)$.

$$
\ln \ln \frac{1}{y}=\left(\frac{E_{a}}{R}\right) \frac{1}{T} ; \quad y=\frac{W_{t}-W_{s e}}{W_{0}-W_{s e}}
$$

Having multiple regions with different slopes, the plots indicated that degradation followed different reaction routes at various orders. The whole degradation region was divided into subdivisions whose boundaries were determined using correlation coefficient values; boundary value was changed until the highest correlation coefficient $\left(r^{2}>0.995\right)$ was obtained for the region under examination. In this way 4 subdivisions were obtained and labeled as I, II, III, and IV for 300-350, 350-400, 400-440, and $440-470^{\circ} \mathrm{C}$; then activation energy estimations were done for each region simultaneously. 
Estimated activation energy values for PVA were found as $86,133,51$, and $84 \mathrm{~kJ} \mathrm{~mol}^{-1}$ for each corresponding section. These values were consistent with the literature cited with small deviations arising from the used method. All samples with an amount of clay content displayed higher values compared to pristine PVA as an indicator of enhanced thermal stability. However, improvement degree was varying with the type of the organoclay used. C-PVA samples displayed a gradual increase related to C-MMT content; activation energy of samples containing 1, 2, 3\% C-MMT by weight in I were calculated as $117,121,126 \mathrm{~kJ} \mathrm{~mol}^{-1}$, while D-PVA samples displayed a better performance compared with C-PVA; the estimated activation energy values were 127, 141, $130 \mathrm{~kJ}$ $\mathrm{mol}^{-1}$ for the same range. The second subdivision, II, where $\mathrm{T}_{1}$ peak located, also showed increased thermal resistance regarding activation energy rise. In this section C-PVA samples granted faintly better results than D-PVA by $7 \mathrm{~kJ} \mathrm{~mol}^{-1}$. The third subdivision, III, displayed similar results for both nanocomposites, an approximate increase of $10 \mathrm{~kJ}$ $\mathrm{mol}^{-1}$ resulted in activation energy estimations of 60-63 $\mathrm{kJ} \mathrm{mol}^{-1}$. Range IV showed that the highest enhancement was obtained for C-PVA with 1\% C-MMT content with an activation energy estimate of $120 \mathrm{~kJ} \mathrm{~mol}^{-1}$, it was followed by D-PVA with 3\% D-MMT content with a value of $111 \mathrm{~kJ} \mathrm{~mol}^{-1}$; remaining samples displayed approximately same result approximately $100 \mathrm{~kJ} \mathrm{~mol}^{-1}$.

These results indicated that interactions among PVA chains and organoclay particles were different in C-PVA and D-PVA nanocomposites. The obtained results were consistent with the literature of PVA with inorganic additive $(15,29)$. It could be deduced that distribution and intercalation of PVA into interlayer spaces of organoclay particles were better for C-PVA than D-PVA; because first sections of the thermal decomposition were related to dehydration and decarboxylation reactions. Thermal degradation was retarded a result of the interactions among organoclay surface and hydroxyl/carbonyl groups of PVA; intercalation degree might affect the magnitude of the retardation because PVA would contain more uniformly dispersed an amount of organoclay to absorb the heat retarding the degradation. In the case of D-PVA, organoclay particles probably dispersed in PVA matrix partly dispersed causing only some parts interacted with PVA; however, organoclay particles were intercalated better for C-PVA resulting in better thermal stability than D-PVA. 

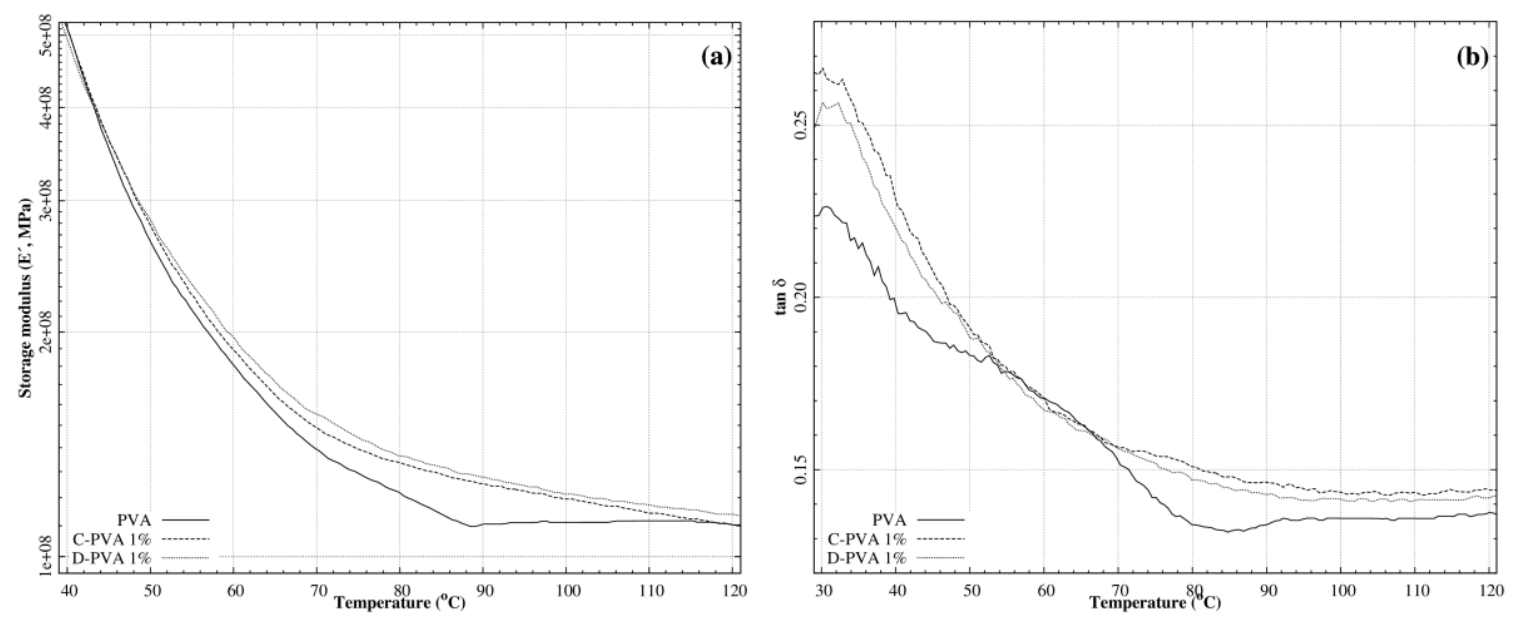

Figure 6. Storage ( $\left.E^{\prime}\right)$ moduli (a) and $\tan \delta(b)$ as a function of temperature for PVA, $1 \%$ C-PVA and D-PVA nanocomposites.

The storage modulus, E', could be used as a measure of the energy stored elastically in the system. Results of dynamic mechanical analyses (DMA) were summarized in Table 2. Introduction of organoclay into PVA matrix also enhanced mechanical strength due to increase in storage moduli of nanocomposites resulting in an increase in elongation regarding organoclay amount (Figure 6a). Each increase in organoclay component of the nanocomposites caused an approximate increase of $15 \%$ elongation of the material. Glass transition temperatures $\left(T_{g}\right)$ were found from peak positions of tan $\delta$ versus temperature plot of each sample; increasing $T_{g}$ values affirmed increase in thermal stability of nanocomposites (Figure $6 b$ ).

Table 2. Results of mechanical properties of PVA and its organoclay nanocomposites

\begin{tabular}{lcccc}
\hline Sample & $\begin{array}{c}\text { Clay } \\
\mathbf{\%}, \mathbf{( w / w )}\end{array}$ & $\begin{array}{c}\text { Tensile } \\
\text { strength } \\
(\mathbf{M p a ,} \mathbf{+ 5})^{\mathbf{a}}\end{array}$ & $\begin{array}{c}\text { Elongation' } \\
(\mathbf{\%}, \mathbf{\pm 2})\end{array}$ & $\left.\mathbf{T}_{\mathbf{g}} \mathbf{(}^{\circ} \mathbf{C}\right)$ \\
\hline PVA & 0 & 110 & - & \\
C-PVA & 1 & 125 & 13 & 30.3 \\
& 2 & 140 & 27 & 32.8 \\
D-PVA & 3 & 153 & 38 & 33.1 \\
& 1 & 127 & 15 & 30.9 \\
& 2 & 136 & 23 & 32.7 \\
\hline
\end{tabular}

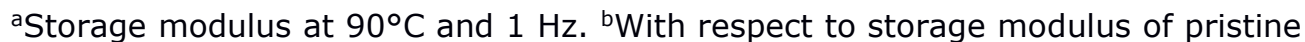
PVA. 'Found from the peak position of $\tan \delta$.

Evaluation of thermal and mechanical analyses indicated that both thermal and mechanical stability of PVA nanocomposites were better than raw PVA polymer even in addition to the smallest amount of clay at $1 \%$ by weight. 


\section{CONCLUSIONS}

Two different types of organoclay were synthesized using cationic surfactants DTABr and $\mathrm{CPBr}$. Spectral and electrokinetic measurements indicated that interactions among clay particles and surfactant mainly occurred at surfaces resulting in surfactant covered clay particles and these interactions also caused an increase along interlayer spacing of clay structure. These organoclays were used to obtain PVA nanocomposites at different proportions of 1,2 , and $3 \%$ by weight. Dispersing C-MMT in PVA matrix with different proportions gave C-PVA nanocomposites while using D-MMT produced D-PVA. Spectral characterizations showed that PVA nanocomposites were obtained with the organoclays. Thermal and mechanical analyses proposed that properties of nanocomposites were enhanced in thermal and mechanical aspects for both nanocomposites concerning pristine PVA. When C-PVA and D-PVA nanocomposites were compared, it was deduced that interactions among PVA chains and organoclays were fairly different.

\section{ACKNOWLEDGMENTS}

This paper is supported by Research Fund of Istanbul Technical University (Project No: 30451) and The Scientific and Technological Research Council of Turkey (TUBITAK) (Project No: 105M088 (MAG-HD-18)).

\section{REFERENCES}

1. Sinha Ray S, Okamoto M. Polymer/layered silicate nanocomposites: a review from preparation to processing. Prog Polym Sci. 2003;28(11):1539-641.

2. Fahmy TYA, Mobarak F. Nanocomposites from natural cellulose fibers filled with kaolin in presence of sucrose. Carbohydr Polym. 2008;72(4):751-5.

3. Bordes P, Pollet E, Averous L. Nano-biocomposites: Biodegradable polyester/nanoclay systems. Prog Polym Sci. 2009;34:125-55.

4. Pavlidou S, Papaspyrides CD. A review on polymer-layered silicate nanocomposites. Prog Polym Sci. 2008;33(12):1119-98.

5. Mallakpour S, Rashidimoghadam S. 8 - Recent developments in the synthesis of hybrid polymer/clay nanocomposites: Properties and applications. In: Hybrid 
Polymer Composite Materials [Internet]. Woodhead Publishing; 2017 [cited 2017 Dec 26]. p. 227-65. Available from: https://www.sciencedirect.com/science/article/pii/B9780081007853000085

6. Utracki LA. Clay-containing polymeric nanocomposites. Shrewsbury: Rapra Technology Ltd; 2004. 2 p.

7. Rhim J-W, Park H-M, Ha C-S. Bio-nanocomposites for food packaging applications. Prog Polym Sci. 2013 Ekim;38(10-11):1629-52.

8. Mermut AR, Lagaly G. Baseline Studies of the Clay Minerals Society Source Clays: Layer-Charge Determination and Characteristics of Those Minerals Containing 2:1 Layers. Clays Clay Miner. 2001;49(5):393-7.

9. Lagaly G, Reese M, Abend S. Smectites as colloidal stabilizers of emulsions I. Preparation and properties of emulsions with smectites and nonionic surfactants. Appl Clay Sci. 1999;14(1-3):83-103.

10. Kokabi M, Sirousazar M, Hassan ZM. PVA-clay nanocomposite hydrogels for wound dressing. Eur Polym J. 2007 Mar;43(3):773-81.

11. Turhan $Y$, Alp ZG, Alkan M, Doğan $M$. Preparation and characterization of poly(vinylalcohol)/modified bentonite nanocomposites. Microporous Mesoporous Mater. 2013;174:144-53.

12. Nistor $\mathrm{M}-\mathrm{T}$, Vasile $\mathrm{C}$. Influence of the nanoparticle type on the thermal decomposition of the green starch/poly(vinyl alcohol)/montmorillonite nanocomposites. J Therm Anal Calorim. 2013 Mar 1;111(3):1903-19.

13. Mehta HR, Murthy ZVP. Preparation of Sodium Montmorillonite Clay Loaded Poly(Vinyl Alcohol)-Chitosan Composite Mixed Matrix Membranes and Their Application in Pervaporation Dehydration of Isopropanol. J Polym Mater New Delhi. 2016 Jun;33(2):319-31.

14. Mallakpour S, Dinari M. Novel bionanocomposites of poly(vinyl alcohol) and modified chiral layered double hydroxides: Synthesis, properties and a morphological study. Prog Org Coat. 2014 Mar;77(3):583-9. 
15. Mallakpour S, Dinari M. Synthesis and Properties of Biodegradable Poly(vinyl alcohol)/Organo-nanoclay Bionanocomposites. J Polym Environ. 2012 Apr $18 ; 20(3): 732-40$.

16. Worrall WE. Clays and ceramic raw materials. London: Elsevier; 1986.

17. Broido A. A simple, sensitive graphical method of treating thermogravimetric analysis data. J Polym Sci Part -2 Polym Phys. 1969;7(10):1761-73.

18. Ünlü $\mathrm{CH}$, Günister $\mathrm{E}$, AtıCl $\mathrm{O}$. Effect of acidity on xylan-montmorillonite bionanocomposites. Mater Chem Phys. 2012;136(2-3):653-60.

19. Vaia RA, Teukolsky RK, Giannelis EP. Interlayer Structure and Molecular Environment of Alkylammonium Layered Silicates. Chem Mater. 1994;6(7):101722.

20. Pan J, Yang G, Han B, Yan H. Studies on Interaction of Dodecyltrimethylammonium Bromide with $\mathrm{Na}$ - and Al-Montmorillonite. J Colloid Interface Sci. 1997 Ekim;194(2):276-80.

21. Chen G, Han B, Yan H. Interaction of Cationic Surfactants with Iron and Sodium Montmorillonite Suspensions. J Colloid Interface Sci. 1998;201(2):158-63.

22. İşçi S, Ece ÖI, Güngör N. Characterization of Rheology, Electrokinetic Properties, and Surface Micromorphology of DTABr-MMT and CPBr-MMT Organoclays. J Compos Mater. 2006;40(12):1105-15.

23. Jayasekara R, Harding I, Bowater I, Christie GBY, Lonergan GT. Preparation, surface modification and characterisation of solution cast starch PVA blended films. Polym Test. 2004;23(1):17-27.

24. Alemdar A, Güngör N, Ece ÖI, Atıcı O. The rheological properties and characterization of bentonite dispersions in the presence of non-ionic polymer PEG. J Mater Sci. 2005;40(1):171-7. 
25. Marel HW van der, Beutelspacher H. Atlas of infrared spectroscopy of clay minerals and their admixtures. Amsterdam: Elsevier Scientific Pub. Co.; 1976.

26. Lai JC-H, Rahman MR, Hamdan S, Liew FK, Rahman MM, Hossen MF. Impact of nanoclay on physicomechanical and thermal analysis of polyvinyl alcohol/fumed silica/clay nanocomposites. J Appl Polym Sci [Internet]. 2015 Nisan [cited 2016 Apr 25];132(15).

Available from:

http://onlinelibrary.wiley.com/doi/10.1002/app.41843/abstract

27. Peng $Z$, Kong LX. A thermal degradation mechanism of polyvinyl alcohol/silica nanocomposites. Polym Degrad Stab. 2007;92(6):1061-71.

28. Gilman JW, VanderHart DL, Kashiwagi T. Thermal Decomposition Chemistry of Poly(vinylalcohol). In: Nelson GL, editor. Fire and Polymers II: Materials and Test for Hazard Prevention. Washington, DC: American Chemical Society; 1994. p. 161-85.

29. Goiti E, Salinas MM, Arias G, Puglia D, Kenny JM, Mijangos C. Effect of magnetic nanoparticles on the thermal properties of some hydrogels. Polym Degrad Stab. 2007;92(12):2198-205. 
